\title{
Pseudomonas aeruginosa Psl Exopolysaccharide Interacts with the Antimicrobial Peptide LG21
}

\author{
Joyce Seow Fong Chin ${ }^{1}$, Sheetal Sinha ${ }^{2,3,4}$, Anjaiah Nalaparaju ${ }^{2}$, Joey Kuok Hoong Yam ${ }^{1}$, \\ Zhiqiang Qin ${ }^{5}$, Luyan Ma ${ }^{6}$, Zhao-Xun Liang ${ }^{2}$, Lanyuan Lu ${ }^{2}$, Surajit Bhattacharjya ${ }^{2}$ and \\ Liang Yang $1,2, *$ it
}

1 Singapore Centre for Environmental Life Sciences Engineering (SCELSE), Nanyang Technological University, Singapore 637551, Singapore; chinsf@ntu.edu.sg (J.S.F.C.); joeyyam@ntu.edu.sg (J.K.H.Y.)

2 School of Biological Sciences, Nanyang Technological University, Singapore 639798, Singapore; SHEETAL003@e.ntu.edu.sg (S.S.); anjai@ntu.edu.sg (A.N.); ZXLiang@ntu.edu.sg (Z.-X.L.); LYLU@ntu.edu.sg (L.L.); surajit@ntu.edu.sg (S.B.)

3 Advanced Environmental Biotechnology Centre, Nanyang Environment and Water Research Institute, Nanyang Technological University, 1 Cleantech Loop, Singapore 637141, Singapore

4 Interdisciplinary Graduate School, Nanyang Technological University, 50 Nanyang Avenue, Singapore 639798, Singapore

5 Departments of Genetics, Louisiana State University Health Sciences Center, Louisiana Cancer Research Center, 1700 Tulane Ave., New Orleans, LA 70112, USA; zqin@lsuhsc.edu

6 State Key Laboratory of Microbial Resources in Institute of Microbiology, Chinese Academy of Sciences (IMCAS), Beijing 100101, China; luyanma27@im.ac.cn

* Correspondence: yangliang@ntu.edu.sg

Received: 9 June 2017; Accepted: 4 September 2017; Published: 16 September 2017

\begin{abstract}
Biofilm formation by opportunistic pathogens serves as one of the major causes of chronic and persistent infections. Bacterial cells in the biofilms are embedded in their self-generated protective extracellular polymeric substances (EPS), which include exopolysaccharides, large adhesin proteins and extracellular DNA. In this study, we identified an antimicrobial peptide (AMP) LG21 that is able to interact specifically with the Psl exopolysaccharide of Pseudomonas aeruginosa, thus it can be used as a diagnostic tool for $P$. aeruginosa biofilms. Molecular dynamics simulation analysis showed that residues numbered from 15 to 21 (WKRKRFG) in LG21 are involved in interacting with Psl. Our study indicates that host immune systems might detect and interact with microbial biofilms through AMPs. Engineering biofilm EPS-targeting AMPs might provide novel strategies for biofilm detection and treatment.
\end{abstract}

Keywords: Pseudomonas aeruginosa; Psl; exopolysaccharide; antimicrobial peptide (AMP); biofilm; EPS

\section{Introduction}

Antimicrobial peptides (AMPs) serve as an essential component of the innate immune system to defend against invading pathogens [1]. AMPs are amphipathic molecules that can directly interact with bacterial cell wall components such as lipopolysaccharide (LPS) and compromise the cell wall integrity [2]. AMPs are also able to target microbial intracellular components such as DNA and RNA [3]. In addition to directly targeting microbial cells, host-derived AMPs are known to modulate the innate immune response and boost the host's capacity for bacterial clearance [4].

Microbial pathogens have successfully evolved multiple strategies to survive from AMP attack. For example, numerous bacterial species have developed AMP sensing mechanisms, which regulate modifications of the cell surface upon AMP exposure [5-7]. Extracellular proteases secreted by bacterial cells have been shown to degrade AMPs and contribute to AMP resistance [8,9]. In addition, microbial 
cells are able to form surface-attached biofilm communities, which represent a distinct lifestyle with increased resistance towards antimicrobials including AMPs [10].

Biofilms consist of microbial cells entrapped by their self-generated extracellular polymeric substance (EPS), such as extracellular DNA, proteins, and exopolysaccharides [11]. EPS serves as a physical shield to protect biofilm cells against harmful conditions such as host immune clearance and antimicrobial treatment. Recently, certain EPS components were shown to interact with bacterial signaling molecules [12] and modulate gene expressions [13]. Biofilm EPS components might interact with AMPs and modulate their functions.

Pseudomonas aeruginosa is an opportunistic pathogen that causes a wide range of nosocomial infections [14]. P. aeruginosa is well-known to form biofilms during infections, which prolong hospitalization and increase the recurrence risk $[15,16]$. The EPS of P. aeruginosa biofilms formed by different strains might contain three exopolysaccharides, alginate, Pel and Psl, which play important roles in biofilm structure maintenance and functions $[17,18]$. Among these three exopolysaccharides, Psl appears to be the most rigid material and crosslinks $P$. aeruginosa cells, leading to microcolony formation [19]. P. aeruginosa small colony variants that over-synthesize Psl have often been observed in clinical settings after acquiring mutations in the wspF gene [20,21]. Psl was shown to protect biofilm cells against antibiotic treatment and phagocytosis $[17,22]$. Previous genetic and biochemical analysis showed that Psl shares conserved structure components (e.g., D-mannose, D-glucose and L-rhamnose) with the LPS [23] and found antibodies to Psl were cross-reactive with LPS [24]. Thus, we hypothesized that AMPs might be able to interact with Psl in a manner similar to LPS.

In this study, we screened a local AMP peptide library to identify AMPs that are able to interact with the P. aeruginosa Psl. We identified an AMP, LG21, that is able to specifically bind Psl. Our study provided evidence that AMPs could be developed as potential biofilm matrix-targeting compounds.

\section{Results and Discussion}

\subsection{LG21 Stains Psl Positive P. Aeruginosa Biofilms}

Psl exopolysaccharide is a critical structural component of $P$. aeruginosa biofilms. We screened biofilms formed by P. aeruginosa wild-type PAO1 (Pel+Psl+), its Psl deficient $\triangle p s l B C D$ mutant (Pel+Psl-), and Pel deficient $\triangle p e l A$ mutant (Pel-Psl+) against our local fluorescent-tagged AMP library. Through this screening, the rhodamine-tagged LG21 was found to strongly stain the Psl+ biofilms (formed by the PAO1 and $\triangle p e l A$ mutant) but not the Psl- biofilm (formed by the $\triangle p s l B C D$ mutant) (Figure 1). Fluorescent signals of the rhodamine-tagged LG21 colocalized well with another well-known Psl stain, TR-ConA [25] (Figure 1). Interestingly, both LG21 and TR-ConA also stain the $\Delta c d r A$ mutant [26], which is unable to produce the Psl-affiliated matrix component CdrA (Figure 1). This result suggests that LG21 might be able to interact with Psl directly. 


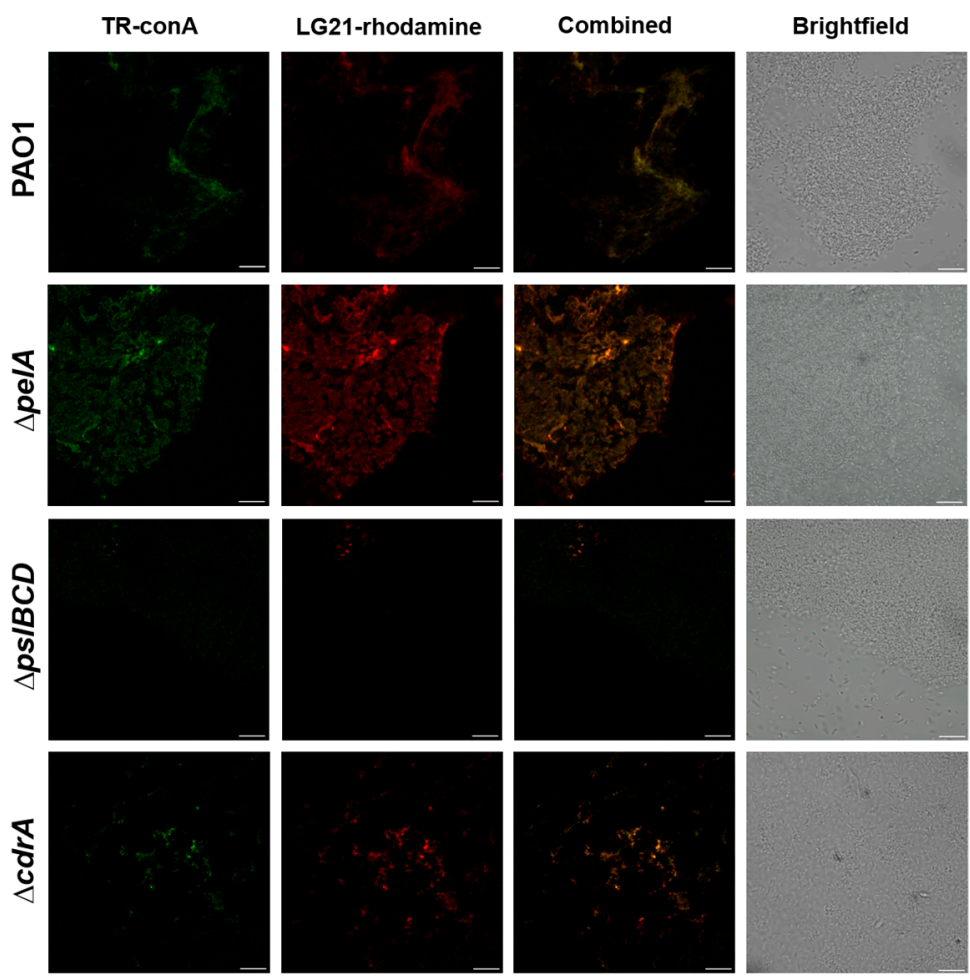

Figure 1. Microscopy images of TR-conA and LG21-rhodamine stained P. aeruginosa wild-type and mutant biofilms. Column 1 depicts the confocal images of the red fluorescence representing the presence of TR-conA. Column 2 depicts the confocal images of the green fluorescence representing the presence of LG21-rhodamine. The merged images are shown in column 3. Bright field images of the biofilms are shown in column 4. Labels of the P. aeruginosa strains are indicated in the left of each row. The experiments were performed in triplicate, and the representative image of each condition is shown as the result. Scale bar, $10 \mu \mathrm{m}$.

\subsection{PslG Treatment Abolishes Binding of LG21 to Psl+ Biofilms}

To further investigate the binding specificity of LG21 to Psl+ P. aeruginosa biofilms, we used rhodamine-tagged LG21 to stain biofilms formed by the WFPA801 strain, a PAO1 derivative strain with an arabinose-inducible $p s l$ promoter [19]. The WFPA801 strain synthesizes Psl in the presence of arabinose in a dose-dependent manner. Rhodamine-tagged LG21 was found to strongly stain the WFPA 801 biofilms cultivated in the presence of $0.5 \%$ arabinose and above and the fluorescent signal colocalized well with TR-ConA (Figure 2). Furthermore, we tested whether treatment of the Psl+ biofilms by using PslG, a glycosyl hydrolase that specifically degrades Psl [27], is able to reduce binding of LG21 to P. aeruginosa biofilms. As we expected, treatment of the WFPA801 biofilms grown at $2 \%$ arabinose by $50 \mathrm{nM}$ PslG for 30 minutes dramatically reduced the binding of Rhodamine-tagged LG21 and TR-ConA to WFPA801 biofilms and a 45-minute treatment by PslG completely abolished the binding (Figure 2). 


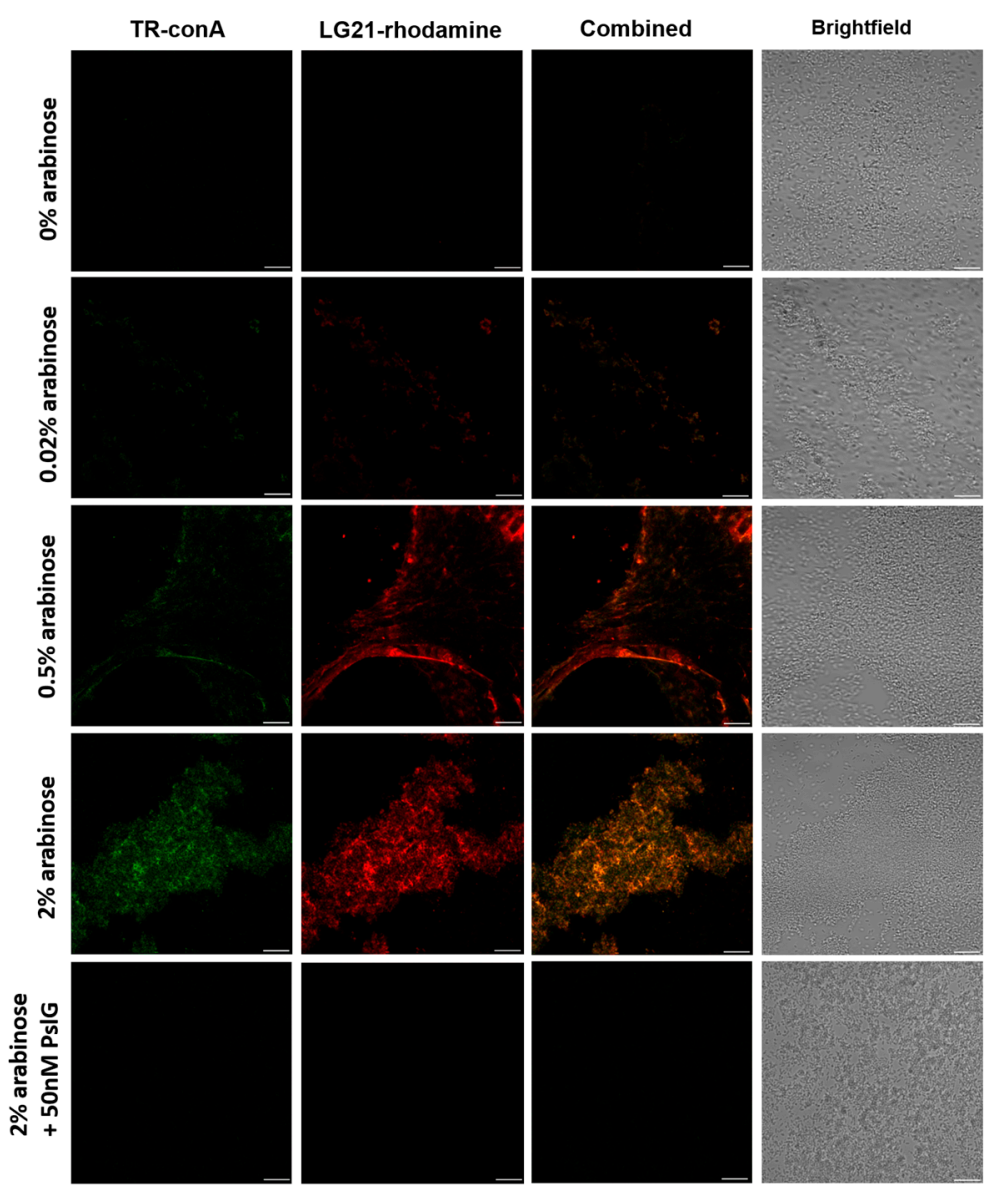

Figure 2. Microscopy images of TR-conA and LG21-rhodamine stained P. aeruginosa WFPA801 biofilms with and without treatment. Column 1 depicts the confocal images of the red fluorescence representing presence of TR-conA. Column 2 depicts the confocal images of the green fluorescence representing presence of LG21-rhodamine. The merged images were shown in Column 3. Bright field images of the biofilms are shown in column 4. Labels of the treatment conditions of P. aeruginosa WFPA801 biofilms are indicated in the left of each row. The experiments were performed in triplicate, and the representative image of each condition is shown as the result. Scale bar, $10 \mu \mathrm{m}$.

\subsection{LG21 Interacts with Crude Extracted Psl Exopolysaccharide}

Next, we investigated whether LG21 interacts with Psl exopolysaccharide by using the crude extracted Psl from the Pel deficient $\triangle p e l A$ mutant (Pel-Psl+) and crude extracted Pel from the Psl deficient $\triangle p s l B C D$ mutant (Pel+Psl-). Since all our P. aeruginosa strains are non-mucoid strains, the production of alginate exopolysaccharide is negligible. We then added LG21 to both crude extracted Psl and Pel, separately, to study the potential interaction between LG21 and exopolysaccharides via fluorescence and NMR methods.

Tryptophan is an excellent intrinsic fluorescent probe to monitor the interactions of peptides with polysaccharides because of its sensitivity towards polarity of the local environment. The fluorescence spectrophotometer data showed that there is a noticeable blue shift of the emission maxima $(\Delta \lambda=8 \mathrm{~nm})$ and quenching of fluorescence when LG21 solution is titrated with Psl (Figure 3a). This indicates that LG21 is in the less polar environment and is interacting with Psl. However, addition of Pel to the LG21 solution does not yield any significant changes (Figure 3a). These results suggest that LG21 preferentially interacts with Psl. 
a

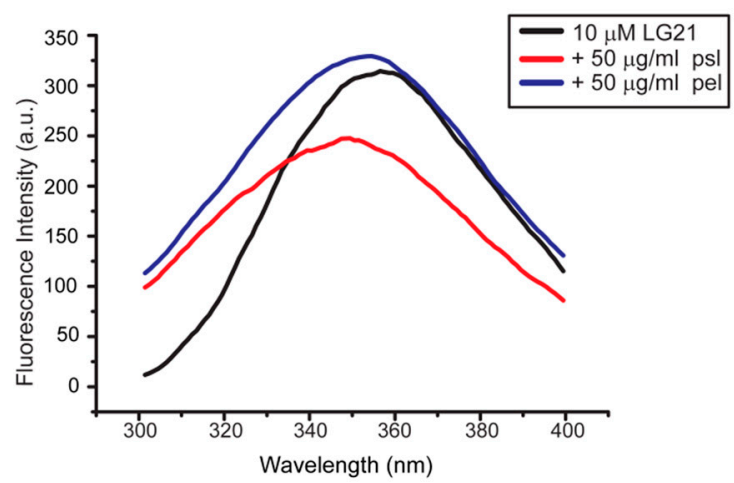

b
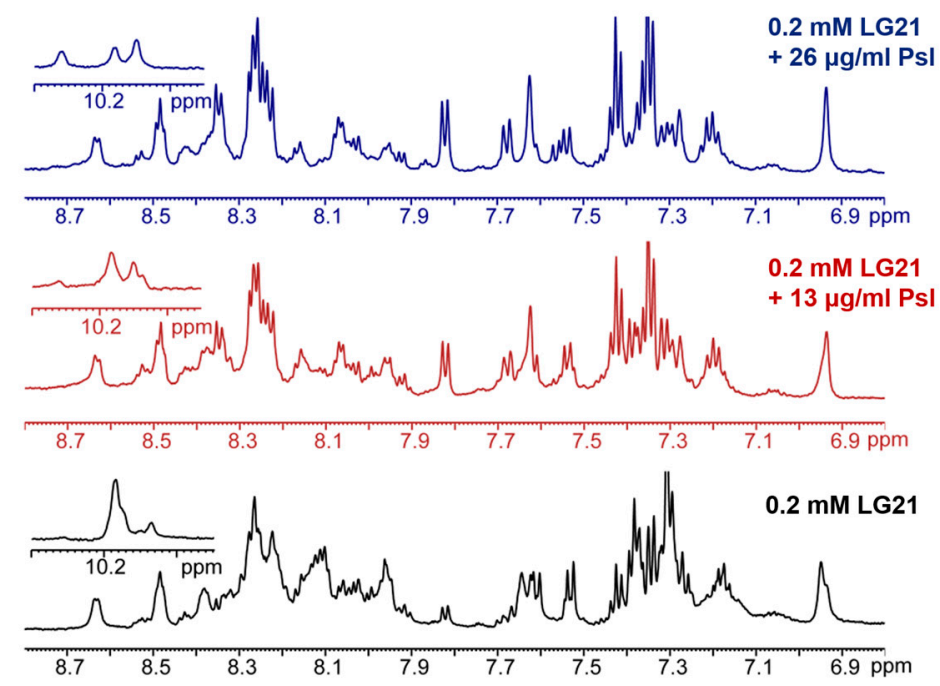

Figure 3. (a) Intrinsic tryptophan fluorescence of LG21 on titrating Pel and Psl recorded by fluorescence spectrophotometer; (b) 1-D NMR spectra of LG21 with and without addition of Psl. Multiple lines at 10.2 ppm suggested more than 1 conformation changes in LG21 upon binding to Psl.

Furthermore, addition of Psl to the LG21 solution showed conspicuous changes in its 1-D NMR spectra (Figure 3b, top) compared to the LG21 control (Figure 3b, bottom), which suggests Psl is able to interact with LG21. Moreover, the tryptophan NMR signal near $10.2 \mathrm{ppm}$ showed multiple lines, indicating that there is more than one conformation of LG21 when in complex with Psl (Figure 3b, top).

\subsection{Molecular Dynamics (MD) Simulation of Psl-LG21 Binding Mode}

To monitor the binding of Psl and LG21, the minimum distance between these two molecules was calculated from the MD simulation trajectory. As shown in Figure S1b (see in the Supplementary Materials), the equilibrium distance between these two units is around $0.2 \mathrm{~nm}$, which indicates the binding of the LG21 peptide to the Psl carbohydrate chain. To obtain more insights into the binding of LG21 to Psl, the minimum distances to each residue in the LG21 from Psl were calculated using the last 150 ns simulation trajectory. As shown in Figure 4a, the residues in LG21 numbered from 15 to 21 (WKRKRFG) consist of the main binding region to the Psl. The interaction energy between the LG21 and Psl polysaccharide were also calculated using the last $150 \mathrm{~ns}$ simulation frames. The contributions from polar and non-polar interactions (e.g., Van der Waals and hydrophobic interactions) in the interaction energy were calculated separately. In the total interaction energy of $\approx-401 \mathrm{~kJ} / \mathrm{mol}$, the contribution from polar interactions is slightly higher $(\approx-223 \mathrm{~kJ} / \mathrm{mol})$ than that of the non-polar interactions $(\approx-178 \mathrm{~kJ} / \mathrm{mol})$. The interaction energies of Psl with individual residues of the LG21 are plotted in Figure S3 along with the error bars. It is observed that the minimum interaction energy 
region in the plot is between the residue numbers 15-21 (WKRKRFG) and also with the residue numbers $7(\mathrm{~N})$ and $10(\mathrm{~K})$, which is consistent with the residues at minimum distances in Figure 4a. These regions are with either charged or polar residues which could initiate the hydrogen bonding with the hydrophilic groups of carbohydrate chain. Although, residues numbered from 15 to 21 (WKRKRFG) in LG21 are interacting more strongly with Psl than the residues 1-14, none of the specific residues has distinguishably high interaction energy. The major conformation of peptide is a random-coil in MD simulations, which could be one reason for having more than one residue interacting with Psl. Moreover, from the individual components of total interaction energy it is confirmed that both polar (hydrogen bonding) and non-polar (Van der Waals and hydrophobic) interactions play significant roles in the binding of LG21 with Psl.

\section{a}

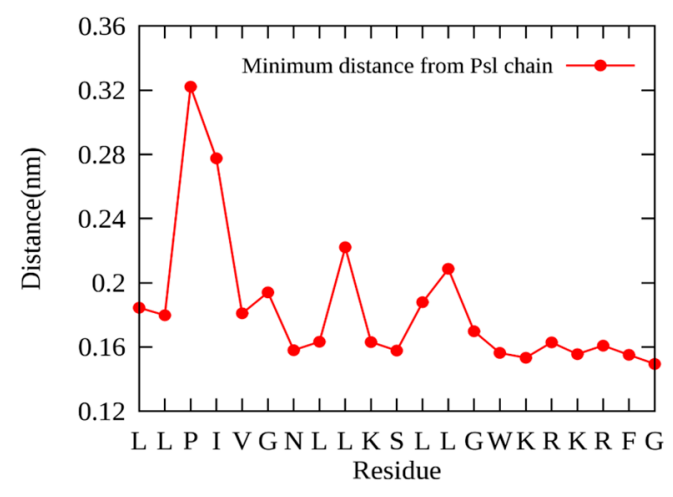

b

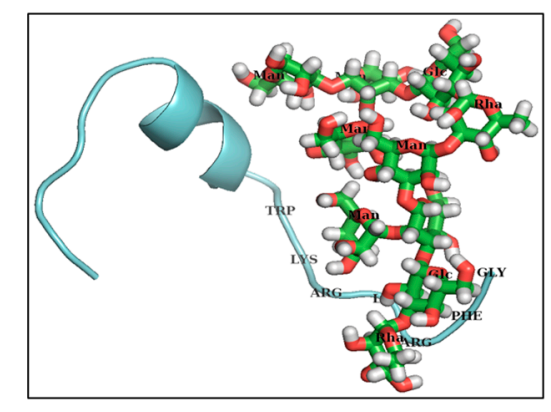

Figure 4. (a) The distribution of minimum distance between the Psl chain and each residue in LG21; (b) Snapshots of the Psl chain and LG21 peptide from the molecular dynamics (MD) simulation at $t=200 \mathrm{~ns}$.

Figure $4 \mathrm{~b}$ shows the simulation snapshot of the LG21 binding to the Psl at $t=200 \mathrm{~ns}$, and the residues in LG21 numbered from 15 to 21 (WKRKRFG) which are at the minimum distances to the Psl are labeled. Based on the qualitative hydrogen-bond analysis and the observations from the trajectory, it is found that the LG21 peptide (residues 15-21) preferably interacts with the region near to the Rhamnose and Glucose monosaccharide groups at the terminal, compared to the Mannose rich region that is slightly hydrophobic in nature [28]. It is also worth noting that the LG21 peptide underwent a conformational change in its secondary structure by some of the residues forming a helical structure from a completely random-coil initial structure. Three independent MD simulations from different initial conformations resulted in the same binding behavior. Figure $\mathrm{S} 4$ shows the structural evolution of the LG21 peptide when it interacts with the Psl. It is observed that the middle residues (6-14) are the main contributors to the helical conformation. The reason could be that the presence of carbohydrate chain (Psl) in the solution decreases the polar nature of water to form hydrogen bonds with the middle residues and the increasing hydrophobic interactions within the peptide induces the helical structure, whereas the terminal residues still have a random-coil structure by being exposed to the aqueous solution. The percentage of secondary structure calculated using the total production run of simulation systems with LG21 and Psl in solution, and only LG21 in solution (control simulation) are shown in Table 1. It is observed that in the control simulation, the LG21 peptide is majorly found as a random coil structure. Previous experimental studies based on NMR and CD-spectra also observed that LG21 is a hybrid antimicrobial peptide that exists in a random-coil conformation in aqueous solution [29,30]. From Table 1, it is also observed that for the simulation system with Psl, the percentage of $\alpha$-helix $(\approx 0.26)$ is substantially higher than the one observed in the control simulation $(\approx 0.05)$, which clearly demonstrates that interaction of LG21 with Psl induces the helicity. The forming 
of the helical conformation in the cationic antimicrobial peptides is observed earlier in the literature, in the presence of carbohydrate chains of biofilm [31].

Table 1. Percentage of secondary structures in the LG21 peptide calculated from systems containing Psl and LG21, and only LG21 (control simulation).

\begin{tabular}{lccccc}
\hline & Coil & Bend & Turn & $\alpha$-Helix & 3-Helix \\
\hline LG21 + Ps1 & 0.58 & 0.07 & 0.07 & 0.26 & 0.02 \\
LG21 only & 0.58 & 0.18 & 0.12 & 0.05 & 0.02 \\
\hline
\end{tabular}

\section{Conclusions}

Exopolysacharides are abundant in bacterial biofilms as a key class of EPS component. Due to their structural complexity, exopolysacharides have distinct structural and functional roles in biofilm formation $[17,18]$. Psl exopolysacharide serves as the major EPS component for P. aeruginosa biofilms and confers resistance towards antibiotic treatment and immune clearance [22,32]. Our recent study showed that Psl attenuated the bactericidal effect of reactive oxidative species to P. aeruginosa [33]. The present work showed that Psl is able to interact with AMP LG21. Since AMPs are well known to function as signaling molecules in modulating the host's immunities [34], Psl over-producing clinical P. aeruginosa variants might thus be able to impair host immunity via AMP binding. Our work did not identify AMPs that bind to the Pel exopolysaccharide. However, it will be worth investigating AMPs that can target the Pel in the future as several P. aeruginosa linages only produce Pel while not Psl. Since AMPs and their mimetics are widely developed and used in different fields, our study suggests that engineering AMPs that target specific biofilm matrix components might facilitate development of strategies to detect and eradicate antibiotic resistant biofilms from both environmental and medical settings.

\section{Materials and Methods}

\subsection{Bacterial Strains, Plasmids, Media and Growth Conditions}

Batch cultivation of P. aeruginosa strains was carried out at $37^{\circ} \mathrm{C}$ in ABT minimal medium [35] supplemented with $2 \mathrm{~g} \mathrm{~L}^{-1}$ glucose (ABTG) or $2 \mathrm{~g} \mathrm{~L}^{-1}$ glucose $+2 \mathrm{~g} \mathrm{~L}^{-1}$ casamino acids (ABTGC). When appropriate, the marker selection in P. aeruginosa, $30 \mu \mathrm{g} \mathrm{mL}^{-1}$ gentamicin $(\mathrm{Gm}), 50 \mu \mathrm{g} \mathrm{mL}^{-1}$ tetracycline (Tc), or $200 \mu \mathrm{g} \mathrm{mL}^{-1}$ carbenicillin $(\mathrm{Cb})$ was used. P. aeruginosa slide biofilms were cultivated on the $24 \times 50 \mathrm{~mm}$ Deckgläser microscope cover glass by inoculating 1:100 diluted overnight P. aeruginosa cultures in $50 \mathrm{~mL}$ BD falcon tubes (BD Biosciences, Singapore) that contained a cover glass, with $10 \mathrm{~mL}$ ABTGC medium. The cultures were then incubated at $37^{\circ} \mathrm{C}$ for $24 \mathrm{~h}$.

\subsection{Screening of Psl-Binding AMPs}

For identifying AMPs that bind to P. aeruginosa Psl exopolysaccharide, rhodamine-tagged peptides from a local peptide library were applied against both biofilms formed by P. aeruginosa wild-type PAO1 strain and its isogenic Psl defective $\triangle p s l B C D$ mutant [17]. The Psl binding fluorescent stain Texas Red ${ }^{\circledR}$-conjugated Concanavalin A (TR-ConA) (Molecular Probes, Eugene, OR, USA) was used as a positive control during the screening. The PAO1 and $\triangle p s l B C D$ mutant slide biofilms were developed as described above. The biofilms were washed twice by dipping into a new falcon tube containing sterile $0.9 \% \mathrm{NaCl}$ to remove the planktonic cells prior to staining with $100 \mu \mathrm{g} \mathrm{mL} \mathrm{m}^{-1}$ TR-ConA and 8 $\mu \mathrm{M}$ rhodamine-tagged LG21 for $15 \mathrm{~min}$.

To monitor fluorescence of rhodamine (excitation $485 \mathrm{~nm} /$ emission $562 \mathrm{~nm}$ ) [29], and TR-ConA (excitation $595 \mathrm{~nm} /$ emission $615 \mathrm{~nm}$ ), the cells were imaged using an LSM780 confocal laser scanning microscope (CLSM; Carl Zeiss, Jena, Germany) with 100 $\times$ objective lens and the images were processed using IMARIS software (Bitplane AG, Zurich, Switzerland). Three independent experiments were 
performed in triplicates and representative images were shown. Rhodamine-tagged AMPs that were able to bind to PAO1 biofilms but not $\triangle p s l B C D$ mutant biofilms were identified and used for further characterization.

\subsection{Binding of LG21 to Psl Overproducing Strain before and after PslG Treatment}

The P. aeruginosa WFPA801 strain that contains an L-arabinose-inducible $p s l$ operon [36] was used to establish biofilms in ABTGC with 0, $0.02 \%, 0.5 \%$ and $2 \%$ L-arabinose at $37^{\circ} \mathrm{C}$ for $24 \mathrm{~h}$. To degrade Psl, $50 \mathrm{nM}$ glycosyl hydrolase PslG was added to 24 hour-old WFPA801biofilms cultivated in ABTGC medium containing 2\% L-arabinose for 0, 30, $45 \mathrm{~min}$. Rhodamine-tagged LG21 and TR-ConA were applied to the treated WFPA801biofilms, followed by CLSM imaging as described above.

\subsection{Crude Extraction of Psl and Pel Exopolysaccharides from P. Aeruginosa}

Pel overproducing $\triangle w s p F \triangle p s l B C D$ strain [13] and the Psl overproducing $\triangle w s p F \Delta p e l A$ strain [13] were used for crude extraction of Psl and Pel exopolysaccharides from $P$. aeruginosa, respectively. Exopolysaccharides were extracted from P. aeruginosa cultures as previously described [17].

\subsection{Tryptophan Fluorescence Spectroscopy}

Intrinsic tryptophan fluorescence of $10 \mu \mathrm{M} \mathrm{LG} 21$ in $10 \mathrm{mM}$ phosphate buffer (pH 7) was measured with a Cary Eclipse fluorescence spectrophotometer (Varian Inc., Palo Alto, CA, USA). The peptide samples were titrated with increasing concentrations of Pel or Psl and fluorescence spectra were recorded with excitation at $280 \mathrm{~nm}$ and emission at $300 \mathrm{~nm}-400 \mathrm{~nm}$.

\subsection{NMR Analysis}

The interaction of LG21 with Psl was studied by performing a series of one-dimensional ${ }^{1} \mathrm{H}$ NMR experiments. LG21 $(0.2 \mathrm{mM})$ in water at a pH 5.5 was titrated with 0,13 and $26 \mu \mathrm{g} / \mathrm{mL}$ Psl, and ${ }^{1} \mathrm{H}$ NMR spectra were recorded at 298K on Bruker DRX $600 \mathrm{MHz}$ NMR Spectrometer (Bruker Scientific Instruments, Billerica, MA, USA).

\subsection{Molecular Dynamics (MD) Simulation of the Interaction between LG21 and Psl Chain}

MD simulations were performed in explicit water to study the interaction of LG21 with Psl. The simulation system to study the interaction of LG21 with Psl was generated by placing one Psl chain containing at least two pentasaccharide repeat units $[\rightarrow 3)-\alpha-\mathrm{L}-\mathrm{RhaP}-(1 \rightarrow 3)-\beta$-DGlcp- $(1 \rightarrow 3)$-[ $\alpha$-D-Manp-( $1 \rightarrow 2)]-\beta$-D-Manp-( $1 \rightarrow 3)-\beta$-D-Manp- $(1 \rightarrow]_{2}$ and one LG21 peptide in a simulation box with charge balancing counterions. At the start of the simulation, the carbohydrate chain and peptide are separated by a distance of $\approx 2 \mathrm{~nm}$ as shown in Figure S1a. All simulations were carried out using GROMACS 4.6.5 simulation package [37]. A control simulation was also performed on a similar system as above but containing only LG21 peptide in the solution for comparison of peptide secondary structure evolution. Additional information on the description of the atomic models of Psl and LG21, force fields used and the details of MD simulations are provided in the supporting information.

Supplementary Materials: The following are available online at www.mdpi.com/2073-4441/9/9/681/s1, Figures S1-S4.

Acknowledgments: This research was supported by the National Research Foundation and the Ministry of Education of Singapore under its Research Centre of Excellence Program. YL is supported by the AcRF Tier 1 (2015-T1-002-100) and AcRF Tier 2 (MOE2014-T2-2-172) from Ministry of Education, Singapore.

Author Contributions: S.B. and L.Y. designed the research. J.S.F.C. and J.K.H.Y. performed the biofilm experiments. A.N. and L.L. performed MD simulation. S.S., Z.Q. and S.B. designed the peptides and performed fluorescence and NMR experiments. L.M. and Z.-X.L. analyzed the data. S.B., L.L. and L.Y. wrote the paper. All authors read and approved the final manuscript.

Conflicts of Interest: The authors declare no conflict of interest. 


\section{References}

1. Pasupuleti, M.; Schmidtchen, A.; Malmsten, M. Antimicrobial peptides: Key components of the innate immune system. Crit. Rev. Biotechnol. 2012, 32, 143-171. [CrossRef] [PubMed]

2. Rosenfeld, Y.; Shai, Y. Lipopolysaccharide (endotoxin)-host defense antibacterial peptides interactions: Role in bacterial resistance and prevention of sepsis. Biochim. Biophys. Acta 2006, 1758, 1513-1522. [CrossRef] [PubMed]

3. Lan, Y.; Ye, Y.; Kozlowska, J.; Lam, J.K.; Drake, A.F.; Mason, A.J. Structural contributions to the intracellular targeting strategies of antimicrobial peptides. Biochim. Biophys. Acta 2010, 1798, 1934-1943. [CrossRef] [PubMed]

4. Alalwani, S.M.; Sierigk, J.; Herr, C.; Pinkenburg, O.; Gallo, R.; Vogelmeier, C.; Bals, R. The antimicrobial peptide 1l-37 modulates the inflammatory and host defense response of human neutrophils. Eur. J. Immunol. 2010, 40, 1118-1126. [CrossRef] [PubMed]

5. $\quad$ Bader, M.W.; Sanowar, S.; Daley, M.E.; Schneider, A.R.; Cho, U.; Xu, W.; Klevit, R.E.; Le Moual, H.; Miller, S.I. Recognition of antimicrobial peptides by a bacterial sensor kinase. Cell 2005, 122, 461-472. [CrossRef] [PubMed]

6. Li, M.; Lai, Y.; Villaruz, A.E.; Cha, D.J.; Sturdevant, D.E.; Otto, M. Gram-positive three-component antimicrobial peptide-sensing system. Proc. Natl. Acad. Sci. USA 2007, 104, 9469-9474. [CrossRef] [PubMed]

7. Fernandez, L.; Jenssen, H.; Bains, M.; Wiegand, I.; Gooderham, W.J.; Hancock, R.E. The two-component system cprrs senses cationic peptides and triggers adaptive resistance in pseudomonas aeruginosa independently of parrs. Antimicrob. Agents Chemother. 2012, 56, 6212-6222. [CrossRef] [PubMed]

8. Thwaite, J.E.; Hibbs, S.; Titball, R.W.; Atkins, T.P. Proteolytic degradation of human antimicrobial peptide 1l-37 by bacillus anthracis may contribute to virulence. Antimicrob. Agents Chemother. 2006, 50, $2316-2322$. [CrossRef] [PubMed]

9. Ulvatne, H.; Haukland, H.H.; Samuelsen, O.; Kramer, M.; Vorland, L.H. Proteases in Escherichia coli and Staphylococcus aureus confer reduced susceptibility to lactoferricin b. J. Antimicrob. Chemother. 2002, 50, 461-467. [CrossRef] [PubMed]

10. Chua, S.L.; Yam, J.K.; Hao, P.; Adav, S.S.; Salido, M.M.; Liu, Y.; Givskov, M.; Sze, S.K.; Tolker-Nielsen, T.; Yang, L. Selective labelling and eradication of antibiotic-tolerant bacterial populations in pseudomonas aeruginosa biofilms. Nat. Commun. 2016, 7, 10750. [CrossRef] [PubMed]

11. Flemming, H.C.; Wingender, J. The biofilm matrix. Nat. Rev. Microbiol. 2010, 8, 623-633. [CrossRef] [PubMed]

12. Seviour, T.; Hansen, S.H.; Yang, L.; Yau, Y.H.; Wang, V.B.; Stenvang, M.R.; Christiansen, G.; Marsili, E.; Givskov, M.; Chen, Y.; et al. Functional amyloids keep quorum-sensing molecules in check. J. Biol. Chem. 2015, 290, 6457-6469. [CrossRef] [PubMed]

13. Chen, Y.; Yuan, M.; Mohanty, A.; Yam, J.K.; Liu, Y.; Chua, S.L.; Nielsen, T.E.; Tolker-Nielsen, T.; Givskov, M.; Cao, B.; et al. Multiple diguanylate cyclase-coordinated regulation of pyoverdine synthesis in pseudomonas aeruginosa. Environ. Microbiol. Rep. 2015, 7, 498-507. [CrossRef] [PubMed]

14. Driscoll, J.A.; Brody, S.L.; Kollef, M.H. The epidemiology, pathogenesis and treatment of pseudomonas aeruginosa infections. Drugs 2007, 67, 351-368. [CrossRef] [PubMed]

15. Bjarnsholt, T. The role of bacterial biofilms in chronic infections. APMIS 2013, 121, 1-58. [CrossRef] [PubMed]

16. Tolker-Nielsen, T. Pseudomonas aeruginosa biofilm infections: From molecular biofilm biology to new treatment possibilities. APMIS 2014, 122, 1-51. [CrossRef] [PubMed]

17. Yang, L.; Hu, Y.; Liu, Y.; Zhang, J.; Ulstrup, J.; Molin, S. Distinct roles of extracellular polymeric substances in pseudomonas aeruginosa biofilm development. Environ. Microbiol. 2011, 13, 1705-1717. [CrossRef] [PubMed]

18. Chew, S.C.; Kundukad, B.; Seviour, T.; van der Maarel, J.R.; Yang, L.; Rice, S.A.; Doyle, P.; Kjelleberg, S. Dynamic remodeling of microbial biofilms by functionally distinct exopolysaccharides. MBio $2014,5$. [CrossRef] [PubMed]

19. Ma, L.; Conover, M.; Lu, H.; Parsek, M.R.; Bayles, K.; Wozniak, D.J. Assembly and development of the pseudomonas aeruginosa biofilm matrix. PLoS Pathog. 2009, 5, e1000354. [CrossRef] [PubMed]

20. Haussler, S.; Tummler, B.; Weissbrodt, H.; Rohde, M.; Steinmetz, I. Small-colony variants of pseudomonas aeruginosa in cystic fibrosis. Clin. Infect. Dis. 1999, 29, 621-625. [PubMed] 
21. Haussler, S.; Ziegler, I.; Lottel, A.; von Gotz, F.; Rohde, M.; Wehmhohner, D.; Saravanamuthu, S.; Tummler, B.; Steinmetz, I. Highly adherent small-colony variants of pseudomonas aeruginosa in cystic fibrosis lung infection. J. Med. Microbiol. 2003, 52, 295-301. [CrossRef] [PubMed]

22. Mishra, M.; Byrd, M.S.; Sergeant, S.; Azad, A.K.; Parsek, M.R.; McPhail, L.; Schlesinger, L.S.; Wozniak, D.J. Pseudomonas aeruginosa psl polysaccharide reduces neutrophil phagocytosis and the oxidative response by limiting complement-mediated opsonization. Cell. Microbiol. 2012, 14, 95-106. [CrossRef] [PubMed]

23. Byrd, M.S.; Sadovskaya, I.; Vinogradov, E.; Lu, H.; Sprinkle, A.B.; Richardson, S.H.; Ma, L.; Ralston, B.; Parsek, M.R.; Anderson, E.M.; et al. Genetic and biochemical analyses of the pseudomonas aeruginosa psl exopolysaccharide reveal overlapping roles for polysaccharide synthesis enzymes in psl and lps production. Mol. Microbiol. 2009, 73, 622-638. [CrossRef] [PubMed]

24. Kocharova, N.A.; Knirel, Y.A.; Shashkov, A.S.; Kochetkov, N.K.; Pier, G.B. Structure of an extracellular cross-reactive polysaccharide from pseudomonas aeruginosa immunotype 4. J. Biol. Chem. 1988, 263, 11291-11295. [PubMed]

25. Tran, C.S.; Rangel, S.M.; Almblad, H.; Kierbel, A.; Givskov, M.; Tolker-Nielsen, T.; Hauser, A.R.; Engel, J.N. The pseudomonas aeruginosa type iii translocon is required for biofilm formation at the epithelial barrier. PLoS Pathog. 2014, 10, e1004479. [CrossRef] [PubMed]

26. Borlee, B.R.; Goldman, A.D.; Murakami, K.; Samudrala, R.; Wozniak, D.J.; Parsek, M.R. Pseudomonas aeruginosa uses a cyclic-di-gmp-regulated adhesin to reinforce the biofilm extracellular matrix. Mol. Microbiol. 2010, 75, 827-842. [CrossRef] [PubMed]

27. Yu, S.; Su, T.; Wu, H.; Liu, S.; Wang, D.; Zhao, T.; Jin, Z.; Du, W.; Zhu, M.J.; Chua, S.L.; et al. Pslg, a self-produced glycosyl hydrolase, triggers biofilm disassembly by disrupting exopolysaccharide matrix. Cell Res. 2015, 25, 1352-1367. [CrossRef] [PubMed]

28. Janado, M.; Yano, Y. Hydrophobic nature of sugars as evidenced by their differential affinity for polystyrene gel in aqueous-media. J. Solut. Chem. 1985, 14, 891-902. [CrossRef]

29. Mohanram, H.; Bhattacharjya, S. Resurrecting inactive antimicrobial peptides from the lipopolysaccharide trap. Antimicrob. Agents Chemother. 2014, 58, 1987-1996. [CrossRef] [PubMed]

30. Mohanram, H.; Bhattacharjya, S. 'Lollipop'-shaped helical structure of a hybrid antimicrobial peptide of temporin b-lipopolysaccharide binding motif and mapping cationic residues in antibacterial activity. Biochim. Biophys. Acta 2016, 1860, 1362-1372. [CrossRef] [PubMed]

31. Chan, C.; Burrows, L.L.; Deber, C.M. Helix induction in antimicrobial peptides by alginate in biofilms. J. Biol. Chem. 2004, 279, 38749-38754. [CrossRef] [PubMed]

32. Billings, N.; Millan, M.; Caldara, M.; Rusconi, R.; Tarasova, Y.; Stocker, R.; Ribbeck, K. The extracellular matrix component psl provides fast-acting antibiotic defense in pseudomonas aeruginosa biofilms. PLoS Pathog. 2013, 9, e1003526. [CrossRef] [PubMed]

33. Chua, S.L.; Ding, Y.; Liu, Y.; Cai, Z.; Zhou, J.; Swarup, S.; Drautz-Moses, D.I.; Schuster, S.C.; Kjelleberg, S.; Givskov, M.; et al. Reactive oxygen species drive evolution of pro-biofilm variants in pathogens by modulating cyclic-di-gmp levels. Open Biol. 2016, 6, 160162. [CrossRef] [PubMed]

34. Brown, K.L.; Hancock, R.E. Cationic host defense (antimicrobial) peptides. Curr. Opin. Immunol. 2006, 18, 24-30. [CrossRef] [PubMed]

35. Chua, S.L.; Liu, Y.; Yam, J.K.; Chen, Y.; Vejborg, R.M.; Tan, B.G.; Kjelleberg, S.; Tolker-Nielsen, T.; Givskov, M.; Yang, L. Dispersed cells represent a distinct stage in the transition from bacterial biofilm to planktonic lifestyles. Nat. Commun. 2014, 5, 4462. [CrossRef] [PubMed]

36. Ma, L.; Jackson, K.D.; Landry, R.M.; Parsek, M.R.; Wozniak, D.J. Analysis of pseudomonas aeruginosa conditional psl variants reveals roles for the psl polysaccharide in adhesion and maintaining biofilm structure postattachment. J. Bacteriol. 2006, 188, 8213-8221. [CrossRef] [PubMed]

37. Pronk, S.; Pall, S.; Schulz, R.; Larsson, P.; Bjelkmar, P.; Apostolov, R.; Shirts, M.R.; Smith, J.C.; Kasson, P.M.; van der Spoel, D.; et al. Gromacs 4.5: A high-throughput and highly parallel open source molecular simulation toolkit. Bioinformatics 2013, 29, 845-854. [CrossRef] [PubMed]

(C) 2017 by the authors. Licensee MDPI, Basel, Switzerland. This article is an open access article distributed under the terms and conditions of the Creative Commons Attribution (CC BY) license (http://creativecommons.org/licenses/by/4.0/). 